\title{
Computer Image Retracking Assessment Based on Pixel Tracking
}

\author{
Yonghong Luo \\ Yiwu Industrial \& Commercial College, Yiwu 322000, China \\ Yonghll322@126.com
}

\begin{abstract}
Computer image retracking is a classical difficult problem in computer image processing. The development of computer image retracking techniques not only influences on other techniques in computer image processing, but also plays an important role in the analysis of biology computer image. Traditional computer image retracking techniques have many defects for complex computer image retracking. Most exiting computer image quality assessment approaches evaluate the difference between computer images of the same size and are not suitable for computer image retracking. In this paper, we propose a novel Content Aware Assessment Metric based on pixel tracking to access the quality of computer image retracking. This metric evaluates the similarity between a retargeted computer image and the original regardless of the difference in computer image size or aspect ratio. By tracking the position of every pixel from the original computer image to the retargeted, including both cumulative distance change and cumulative angle change to measure the local difference between the two computer images. By comparing our experimental results with user surveys, we demonstrate that the proposed assessment metric is consistent with human experience.
\end{abstract}

Keywords: Computer image retracking, Pixel tracking, Assessment metric

\section{Introduction}

In recent years, computer image retracking became more and more important and popular because it enables the same computer images (or video to be displayed) on various screens of different resolutions and sizes such as smart phones and tablets, cameras and camcorders, or TVs and projectors. Therefore, how to assess the quality of the retracking methods comes to be a new challenge for researchers interested in computer version.

However, most exiting computer image quality assessment approaches evaluate the difference between computer images of the same size. The direct application of such a method is not suitable for computer image retracking because the retargeted computer image is different from the original in size and/or aspect ratio.

In this paper, we propose a novel assessment metric, based on pixel tracking in computer image retracking. This assessment metric can evaluate the similarity of the retargeted computer image and the original computer image in both local and global information. The local difference calculated by tracking the position of every pixel from the original computer image to the retargeted and summing up the cumulative distance change and cumulative angle change. While the global change is assessed by taking into consideration a variation of the total energy in the computer image is in the assessment metric. Experiments and user surveys demonstrate that the assessment resulting from this metric is consistent with human observation. 


\section{Related Work}

Content-aware computer image retracking techniques can adapt the computer images into various size and/or aspect ratios to fit in different screens or panel windows. Because information is not $100 \%$ preserved during computer image retracking, quality of the retargeted computer images is of great concern to user experience. Therefore, an assessment metric of both theoretic soundness and practical effectiveness is critical for the design and implementation of computer image retracking algorithms and applications.

There are subjective and objective assessment methods to evaluate the quality of retargeted computer images. Subjective assessment is obtained from statistics of human evaluations, by conducting user surveys or studying human responses to the computer images.

Castllo et al., [Castillo, 11] collected eye-tracking data on a number of computer images from dozens of observers and use it as ground truth data to analyze possible changes in regions of interest due to retracking. Sun and Ling [Sun, 13] proposed a new thumbnailing framework and three types of user studies and they evaluate the quality of the thumbnailing by adding up the subjective evaluation results of the observers. Such subjective assessment methods can reflect the preferences of the viewer, which could be an advantage or disadvantage. However, conducting this type of assessment, such as the mean opinion scores metric, is always time-consuming and expensive.

An objective assessment using computational models to measure the perceptual quality of computer images is therefore much desired. Objective assessment has been intensively studied and the methods of this type are further classified into full reference (FR), reduced reference (RR) and non-reference (NR) approaches. FR methods require full information of the original image as references while RR methods only require partial information of original images for quality assessment. NR methods evaluate distorted images in a blind way which is an extremely stiff work. Unfortunately, for both FR and RR methods, the original image and the distorted image are required to be in the same size, and it is obvious that both the two methods are not suitable for image retracking assessment. Moreover, if an NR method is applied to image retracking, the information of the original image will be completely discarded and the assessment may be inaccurate.The method we provide in this paper is a FR assessment method for evaluating the quality of retargeted computer images by using a novel error metric to measure the difference between the retargeted computer image and the original.

Liu et al., [Liu, 11] proposed an objective method that organizes in a top-down manner the computer image features from global to local viewpoints. On the one hand they designed a scale-space matching method to facilitate extraction of global geometric structures from retargeted computer images. On the other hand they established local pixel correspondence by traversing the scale space from coarse levels to fine levels. And an objective assessment metric is then built based on both global geometric structures and local pixel correspondence. Dong et al., [Dong, 09] and Hua et al., [Hua, 11] introduced Bidirectional Similarity Measure into seam carving [Avidan, 07] [Rubinstein, 08] where they achieve the retargeted computer images by setting a threshold computed via Bidirectional Similarity Measure to guide the retracking process. However, these methods need to divide the original computer image and the retargeted computer image into computer image blocks of the same size, and the distance between blocks in the original computer image and the retargetred must be computed after each seam is deleted or inserted. This would lead to a result of a large amount of computation and low efficiency. The assessment method of $\mathrm{Wu}$ et al., [Wu, 13] is highly dependent on the important map of the computer image achieved by Itti's saliency detector [Itti, 01]. The distance of the important area in the retargeted computer image and the original computer image is computed. However, if the saliency detector cannot catch the important area in the computer image, the assessment results will not be convincing. In addition, the degree of 
deformation in the context region of the important area should also be considered because the global vision of the image may be destroyed in the retargeted image.

\section{Assessment Metric Based on Pixel Tracking}

Considering that users are often sensitive to the change of the color, our scheme first segments an computer image to multiple regions by Mean Shift method [Comaniciu, 02]. An example of the segmenting result is showed in Figure 1 (c), and (d). Following this step, the scheme evaluates the change of every region in the retargeted computer image by comparing to the original computer image. Through this procedure, the position change of each pixel in the retargeted computer image is considered.

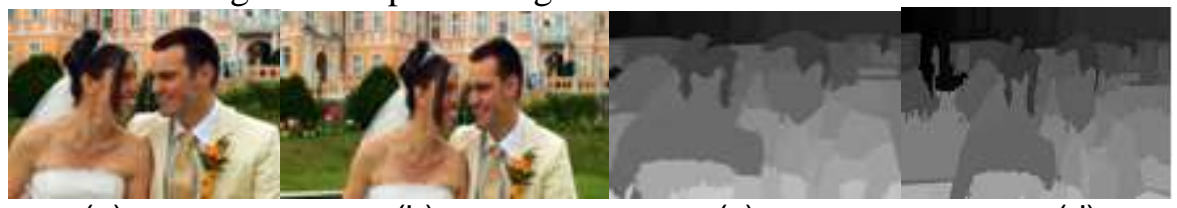

(a)

(b)

(c)

(d)

Figure 1. The Original Computer Image (a) is Retargeted to $75 \%$ Width by Seam Carving and the Retargeted Computer Image is Showed in (b). The Segmenting Results by Mean Shift for (a) and (b) are Showed in (c) and (d).

\subsection{Pixel Tracking}

Suppose that the original computer image $I$ is divided into $p$ regions represented as $R$ $=\left\{R_{1}, R_{2}, \ldots, R_{\mathrm{p}}\right\}$ by Mean Shift method. We use a four-dimensional array $\left(x, y, R_{i}, n_{u}\right)$ $(i=1,2, \ldots, p)$ to denote the coordinate of each pixel in the computer image where $x$ is the row coordinate and $y$ is the column coordinate of the pixel. In order to track the pixel in the original computer image, we give each pixel a uniform number $n_{u}$ according to its order in the image from top to bottom and from left to right. That is, for an image of size $m \times n, n_{u}=x+n(y-1)$. Then, the number $n_{u}$ will be a integer between 1 and $m \times n$.

After retracking computer image $I$ to the resulting computer image $I^{\prime}$ by seam carving, we use $r_{i}$ to trace the corresponding region $R_{i}$ in $I^{\prime}$. Let the regions remained in the retargeted computer image $I^{\prime}$ be $r=\left\{r_{1}, r_{2}, \ldots, r_{q}\right\}$ where $q \leq p$ and the new coordinates of each pixel in computer image $I^{\prime}$ can be $\left(x^{\prime}, y^{\prime}, r_{i}, n_{u}\right)(i=1,2, \ldots, q)$. Note that some points in $R_{i}$ are carved in $r_{i}$, and some region $R_{i}$ may even no longer exist in $I^{\prime}$. In order to consider the position change of each pixel in the retargeted computer image, we conversely track the pixel coordinate in the original computer image based on the uniform number $n_{u}$. In this way, the pixels that are carved out don't need to be considered any more.

\subsection{Content Aware Assessment Metric}

We concern not only the important regions as discussed in [Wu, 13] but also the global visual effect that is more sensitive to general human viewers. Therefore, we take into consideration the changes in each and every region $r_{1}, r_{2}, \ldots, r_{q}$. Then we randomly sample a large number of points from each region $r_{i}(i=1,2 \ldots q)$ in $I^{\prime}$ and, for each pair of sampling points, evaluate the change of the following two measures before and after seam carving. I.e., the distance between the two points and the angle between the x-axis and the line segment connecting the two points. Moreover, we consider the energy change of the retargeted computer image comparing to the original computer image. 
3.2.1 Distance Change Metric: Suppose we label a pair of two points in region $r_{i}$ of the retargeted computer image I' as $A_{j}$ and $B_{j}$, and the two points corresponding to them in the original computer image $I$ as $A_{j 0}, B_{j 0}$. The distance change of the two points $A_{j}$ and $B_{j}$ comparing to their corresponding points $\mathrm{A}_{j 0}$ and $\mathrm{B}_{\mathrm{j} 0}$ is shown in Figure 2.

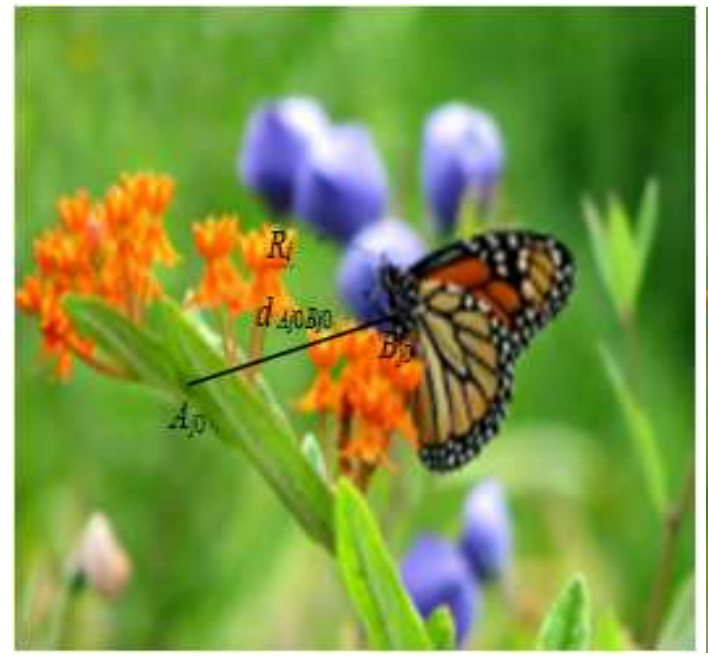

(a)

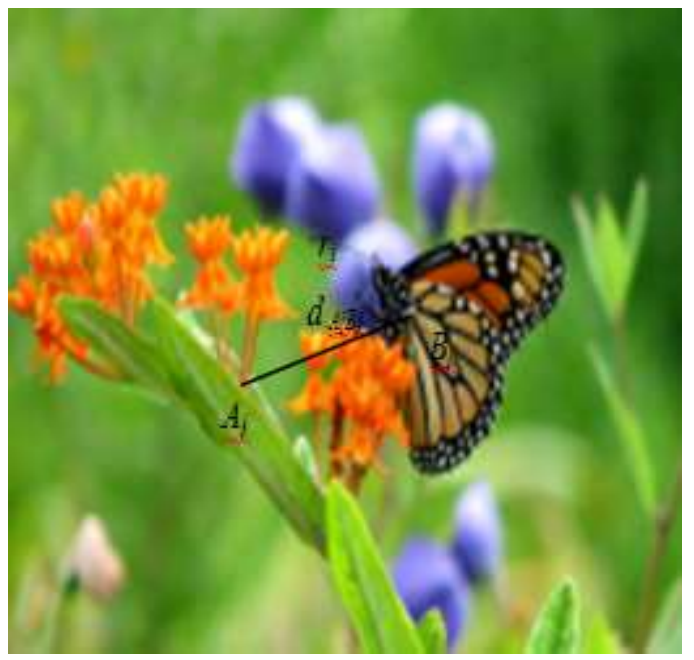

(b)

Figure 2. Two Points in Region $r_{i}$ of l' are Labeled as $A_{j}, B_{j}$ and the Two Original Points Corresponding to them in I as $A_{j 0}, B_{j 0}$.

In order to control the magnitude of the distance change value, we normalized the distance change to be:

$d_{s j}=\left|\frac{d_{A_{j} B_{j}}-d_{A_{j 0} B_{j 0}}}{d_{A_{j 0} B_{j 0}}}\right|$,

where $d_{A_{j} B_{j}}$ is the new distance of the two points in the retargeted computer image $I^{\prime}$ and $d_{A_{j 0} B_{j 0}}$ is their original distance in the original computer image $I$.

Considering that different pixels have different levels of importance in the image, we further compute the importance of each point using gradient magnitude and accordingly assign a weight to the distance change $d_{s j}$. The weight is $e_{A_{j 0}}+e_{B_{j 0}}$, where $e_{A_{j 0}}$ and $e_{B_{j 0}}$ are the importance values of point $A_{j 0}$ and $B_{j 0}$ respectively. The importance value of the pixel is computed using its gradient magnitude defined as:

$e(I(x, y))=\left|\frac{\partial}{\partial x} I(x, y)\right|+\left|\frac{\partial}{\partial y} I(x, y)\right|$

where $I$ is an image and $(x, y)$ is the coordinate of the pixel.

Therefore, the (weighted) distance change of the two points $A_{j}$ and $B_{j}$ comparing to their corresponding points $A_{j 0}$ and $B_{j 0}$ is defined as:

$d_{s j}{ }^{\prime}=\left(e_{A_{j 0}}+e_{B_{j 0}}\right) \cdot\left|\frac{d_{A_{j} B_{j}}-d_{A_{j 0} B_{j 0}}}{d_{A_{j 0} B_{j 0}}}\right|$. 
In order to measure the change of the region $r_{i}$ of $I^{\prime}$ comparing to the corresponding region in the original computer image $I$, we randomly sample $w$ pairs of points in region $r_{i}$ and then compute the cumulative distance change of these $w$ pairs of points as:

$$
D_{i}=\frac{\sum_{j=1}^{w}\left(e_{A_{j 0}}+e_{B_{j 0}}\right) \cdot\left|\frac{d_{A_{j} B_{j}}-d_{A_{j 0} B_{j 0}}}{d_{A_{j 0} B_{j 0}}}\right|}{\sum_{j=1}^{w}\left(e_{A_{j 0}}+e_{B_{j 0}}\right)} \quad(i=1,2, \cdots p)
$$

Finally, the distance change in all regions in the retargeted computer image will be the summation of the distance change in every region, which is defined as:

$$
D_{\text {sum }}=\sum_{i=1}^{p} D_{i} \quad(i=1,2, \cdots p) .
$$

3.2.2. Angle Change Metric: We also consider computing the angle change between the horizontal axis and the connection of the two points $A_{j}$ and $B_{j}$ comparing to their corresponding points $A_{j 0}$ and $B_{j 0}$ in the original computer image (See Figure 3 ). The angle $\theta_{A_{j} B_{j}}$ between the horizontal axis and the connection of the two points $A_{j}$ and $B_{j}$ in the retargeted computer image is showed in Figure 3 (b).

Using the same formalization and weight as for the distance change, we define, the angle change of the two points $A_{j}$ and $B_{j}$ comparing to their corresponding points $A_{j 0}$ and $B_{j 0}$ as follows.

$$
\theta_{s j}{ }^{\prime}=\left(e_{A_{j 0}}+e_{B_{j 0}}\right) \cdot\left|\frac{\theta_{A_{j} B_{j}}-\theta_{A_{j 0} B_{j 0}}}{\theta_{A_{j 0} B_{j 0}}}\right| .
$$

Similar to the cumulative distance change, the cumulative angle change of $w$ pairs of points in region $r_{i}$ is normalized as:

$$
A g_{i}=\frac{\sum_{j=1}^{w}\left(e_{A_{j 0}}+e_{B_{j 0}}\right) \cdot\left|\frac{\theta_{A_{j} B_{j}}-\theta_{A_{j 0} B_{j 0} 0}}{\theta_{A_{j 0} B_{j 0}}}\right|}{\sum_{j=1}^{w}\left(e_{A_{j 0}}+e_{B_{j 0}}\right)} \quad(i=1,2, \cdots p),
$$

and the summation of the angle change in all regions of the retargeted computer image is:

$$
A g_{\text {sum }}=\sum_{i=1}^{p} A g_{i} \quad(i=1,2, \cdots p)
$$




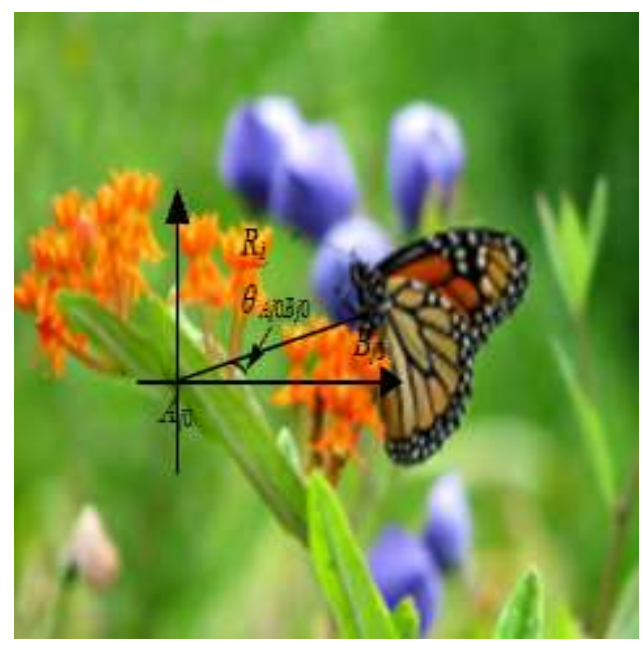

(a)

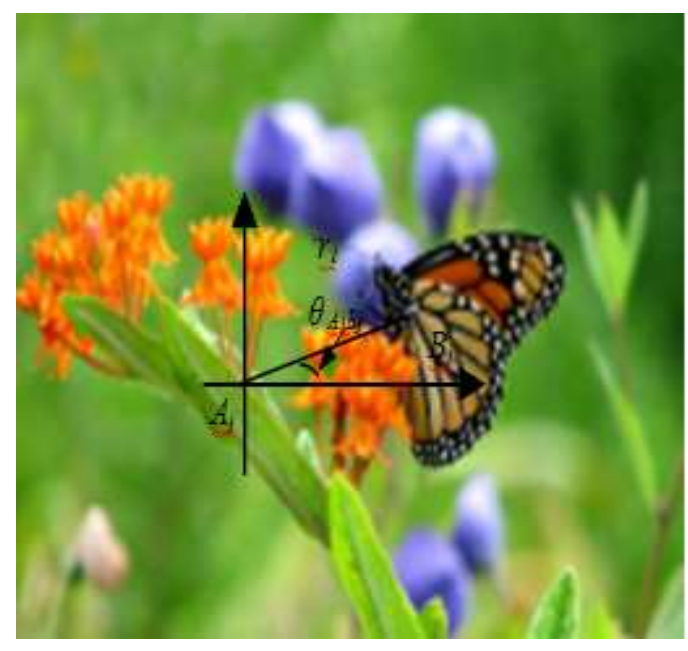

(b)

Figure 3. The Angle $\theta_{A_{i} B_{B}}$ between the Horizontal Axis and the Line Segment Connecting the Two Points $A_{j}$ and $B_{j}$ in the Retargeted Computer Image is Showed in (b), While the Corresponding Angle in the Original Computer Image is Showed in (a)

3.2.3. Energy Change Ratio: As mentioned above, we are concerned about the global visual effect of the retargeted computer image. Therefore, we also add to the metric a measure of the retention of the feature points in the original computer image. Indeed, we compute the importance of each pixel in the original computer image and use it as a basis to measure the remaining feature information in the retargeted computer image.

When an computer image is downsized, the pixel of high importance value should be preserved in the retargeted computer image. The more high importance pixels are retained, the more satisfactory the retargeted computer image is. Let $e\left(I^{\prime}\right)$ and $e(I)$ be the energy of the retargeted computer image and the original computer image respectively. The energy change ratio of the retargeted computer image comparing to the original one is defined as:

$R_{a t}=\frac{e\left(I^{\prime}\right)}{e(I)}$, and $R a t_{e} \in(0,1)$

When an computer image is enlarged, new pixels are added to the retargeted computer image so that $R a t_{e} \geq 1$. However, we don't want to add pixels of high importance value, meaning that $R a t_{e}$ approaching to 1 is the best result.

3.2.4. Assessment Metric: Finally, we define the Content Aware Assessment Metric to be the linear combination of the three measures described above. When an computer image is downsized, the assessment metric is defined as follows:

$E M=D_{\text {sum }}+A g_{\text {sum }}+\frac{1}{R a t_{e}}$

and when an computer image is enlarged, the assessment metric is:

$E M=D_{\text {sum }}+A g_{\text {sum }}+R a t_{e}$.

When an computer image is retargeted, the smaller its EM value is, the better it looks because more important regions have been preserved. On the contrary, the 
computer image would be not satisfactory. In the following Section 4, we will verify this conclusion by means of experiments and user surveys.

Let $A_{i}(l, t)$ is the area of $E_{i}(l, t)$ and $\max _{i \in\{1,2,3,4\}} A_{i}(l, t)$ illustrate the maximum area of $\left.E_{i}(l, t)\right|_{i \in\{1,2,3,4\}}$. To obtain the optimal object detection under $\kappa(l, t)=1$, the confidence vote $v_{i}(l, t)$ is computed by weighting the area ratio between $A_{C}(l, t)$ and $A_{i}(l, t)$, and the area ratio between $A_{i}(l, t)$ and $\max _{i \in\{1,2,3,4\}} A_{i}(l, t)$ as (12). The optimal object detection $M(\mathbf{x}, l, t)$ is outputted as (13). Columns 5-6 in Figure 4 illustrate the intersection states and the fused optimal detections respectively.

$v_{i}(l, t)=\frac{\alpha \cdot A_{C}(l, t)}{A_{i}(l, t)}+\frac{(1-\alpha) \cdot A_{i}(l, t)}{\max _{i \in\{1,2,3,4\}} A_{i}(l, t)}$

$M(l, t)=E_{i}(l, t) \mid E_{i}(l, t):=\arg \left(v_{i}(l, t)=\max _{i \in\{1,2,3,4\}} v_{i}(l, t)\right)$

To include of both cases (i.e., $\kappa(l, t)=1$ and 0 ), the generalized detection output $O(l, t)$ is described as (14).

$O(l, t)=\kappa(l, t) \cdot M(l, t)+(1-\kappa(l, t)) \cdot F(l, t)$

Individual detection refers to the one-to-one relationship between the object and the bounding box, while merging detection defines the many-to-one relationship between the multi-object and one bounding box. The individual detection ratio is the percentage of the object numbers of individual detection from the total number of valid object detections. Similarly, the merging detection ratio is computed.

$\frac{A_{O(l, t) \cap R(l, t)}}{A_{R(l, t)}} \geq 75 \%$

Due to the scale and area of the merging detection may not satisfy the multi-threshold limitation, the objects corresponding to a same detection bounding box may be regarded as invalid detection. Under this case, the detected valid object numbers under background difference and temporal difference are bound to decrease. To ensure the comparability among three methods, we sum the detected object number of the above two methods without considering multi-threshold limitation. Still, both methods achieve lower detections number than our method in images sequence 1. As shown in Table 1, 254 objects are detected under the proposed method with higher individual detection ratio $92.1 \%$ than other two methods (85.5\% and $81.0 \%$ respectively). Although the detected valid objects number with temporal difference in images sequence 2 is slightly higher than our method, the individual detection ratio $86.7 \%$ is far below the latter $(98.1 \%)$. The merging detections of our method in both image sequences are at the lowest ratios (7.9\% and $1.9 \%$ respectively). It is obvious that the proposed method achieves more accurate and robust detection performance than background difference and temporal difference methods.

\section{Experimental Results}

In general, for a computer image, the more information is deleted, the worse visual effects are. To our assessment metric, the value should gradually increase. We resize the computer images in Figure 4 (a), using seam carving to reduce the width by 100, 200, 300, 
400, 500, 600, 700 and 800 pixels and show results in Figure 4 (b)-(i). The assessment metric value is shown in Figure 5. From these pictures we can see that the result of our Content Aware Assessment Metrics improves as the width of the resized computer image reduces. It is consistent with the subjective observation.
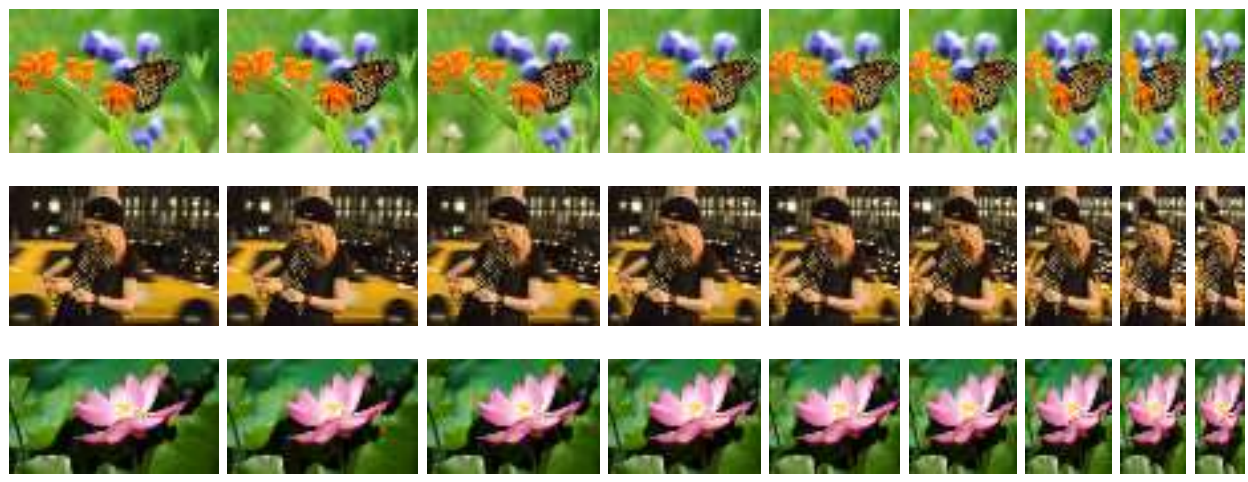

(a)

(b)

(c)

(d)

(e)

(f)

(g) (h) (i)

Figure 4. Resized Images of Butterfly(Img1), DKNYGirl(Img2) and Lotus(Img3) by Removing Different Numbers of Vertical Seams. The Leftmost Column Shows the Original Computer Images and the Following Columns, from (b) to (i), Show the Results of Removing 100, 200, 300, 400, $500,600,700,800$ Vertical Seams by Seam Carving

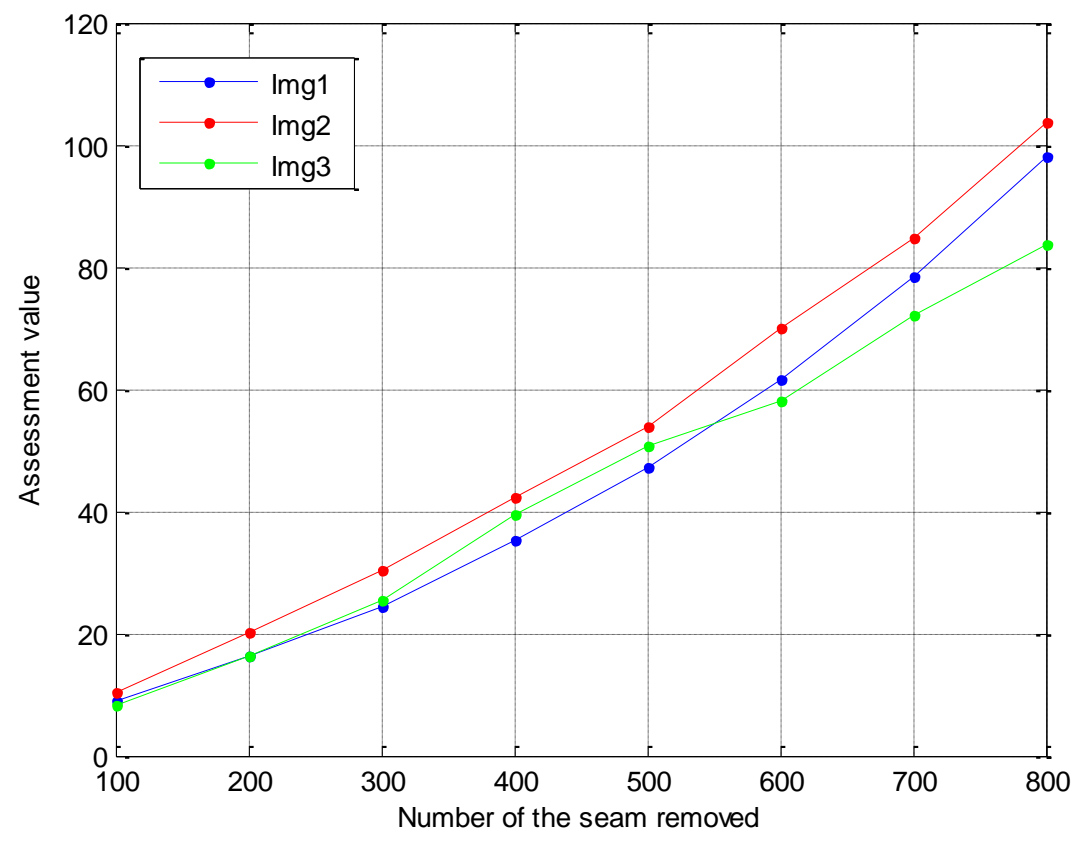

Figure 5. Assessment Values of the Three Computer Images in Figure 4 Using our Content Aware Assessment Metric based on Pixel Tracking

\section{User Surveys}

We further conduct a user surveys to assess the consistency of the proposed perceptual error metric with subjective human evaluation. Total of 50 computer images of RetargetMe dataset [Rubinstein, 10] [Rubinstein, 10]' are used in this survey. Each 
resized computer image is obtained from original computer images using the seam carving method. Total of 30 volunteers of different age, gender, and education background participated in our user surveys. In this survey, participants were instructed to rate the similarity of a resized computer image to the original computer image according to five intervals of the scale: 1-10 (bad), 11-20 (poor), 21-30 (fair), 31-40 (good), and 4150 (excellent). A pair of computer images at full size is simultaneously displayed side by side on the screen, with the original computer image on the left and the retargeted computer image on the right. The 50 computer image pairs were displayed in random order. The objective score calculated by our error metric and the subjective score collected from the user surveys are shown in Figure 6. We can see that the data fits in an exponential function. This result demonstrates that the result of our proposed assessment metric is consistent with the human's subjective observation.

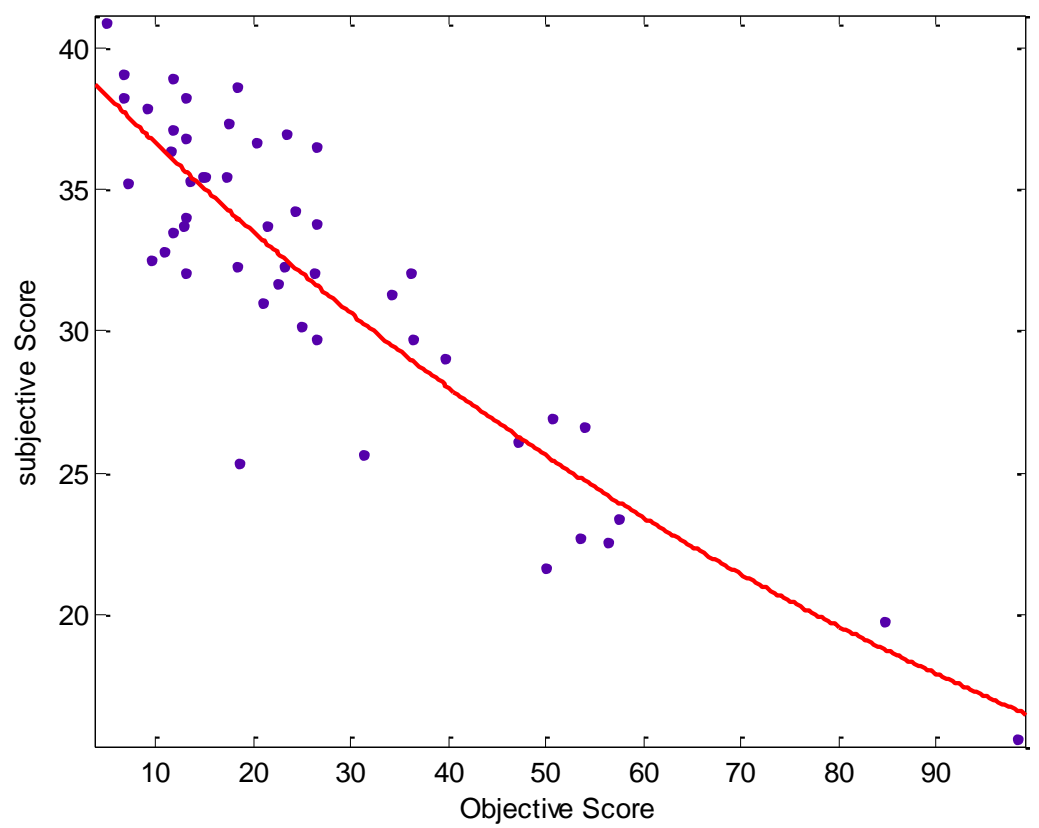

Figure 6. The Corelation of Subjective Scores and Objective Scores

\section{Conclusion}

We have presented a novel Content Aware Assessment Metric based on pixel tracking for computer image retracking. This metric can evaluate the similarity between a retargeted computer image and its original computer image although they have different size and/or aspect ratio. By tracking the new position of every pixel from the original computer image to the retargeted, we build a Content Aware Assessment Metric that combines the numeric measures of cumulative distance change, cumulative angle change, and energy change ratio. In this way, not only are the local difference between the retargeted computer image and the original computer image calculated, the global change in the retargeted computer image is considered as well. Experimental results demonstrate that the result of the proposed assessment metric is consistent with the human's subjective observation and a user surveys shows that our assessment metric comply with human evaluation by randomly chosen non-professional observers. 


\section{References}

[1] S. Avidan and A. Shamir, "Seam carving for content-aware computer image resizing", ACM Trans. Graph., vol. 26, no. 3, (2007), pp. 267-276.

[2] S. Castillo, T. Judd and D. Gutierrez, "Using eye-tracking to assess different computer image retracking methods", In Proceedings of the ACM SIGGRAPH Symposium on Applied Perception in Graphics and Visualization, (2011), pp. 7-14.

[3] D. Comaniciu and P. Meer, "Mean shift: a robust approach toward feature space analysis", IEEE Transactions on Pattern Analysis and Machine Intelligence, vol. 24, no. 5, (2002), pp. 603-619.

[4] W. Dong, N. Zhou, J.-C. Paul and X. Zhang, "Optimized computer image resizing using seam carving and scaling", ACM Trans. on Graphics (Proc. of SIGGRAPH ASIA), vol. 28, no. 5, (2009), pp. 1-10.

[5] S. Hua, X. Li and Q. Zhong, "Similarity criterion for computer image resizing", EURASIP Journal on Advances in Signal Processing, vol 1, (2011), pp. 1-8.

[6] L. Itti and C. Koch, "Computational modelling of visual attention", Nature Reviews Neuroscience, vol. 2, no. 3, (2001), pp. 194-204.

[7] Y. J. Liu, X. Luo, Y. M. Xuan, W. F. Chen and X. L. Fu, "computer image retracking quality assessment”, In Computer Graphics Forum, vol. 30, no. 2, (2011), pp. 583-592.

[8] J. Sun and H. Ling, "Scale and Object Aware computer image Thumbnailing", International journal of computer vision, vol. 104, no. 2, (2013), pp. 135-153.

[9] M. Rubinstein, A. Shamir and S. Avidan, "Improved seam carving for video retracking", ACM Trans. on Graphics, vol. 27, no. 3, (2008), pp. 1-9.

[10] M. Rubinstein, D. Gutierrez, O. Sorkine and A. Shamir, "A comparative study of computer image retracking", In ACM transactions on graphics (TOG), vol. 29, no. 6, (2010), pp. 160.

[11] RetargetMe. http://people.csail.mit.edu/mrub/retargetme/

[12] L. Wu, L. Cao, J. Wang and S. Liu, "Content Aware Metric for computer image Resizing Assessment", The Era of Interactive Media, (2013), pp. 255-265.

\section{Author}

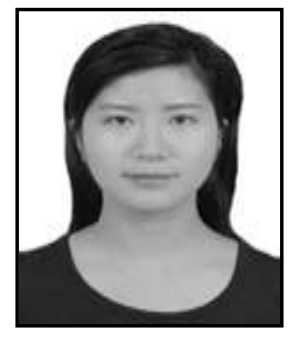

Yonghong Luo, assisted professor, Research direction: computer image; computer image analysis. 